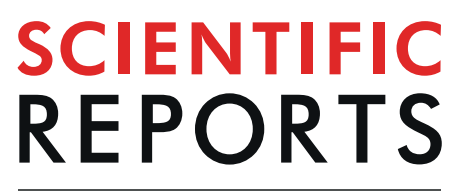

natureresearch

\title{
Late Gadolinium Enhancement Predicts Improvement in Global Longitudinal Strain after Aortic Valve Replacement in Aortic Stenosis
}

\author{
Tsuyoshi Fujimiya ${ }^{1,2^{*}}$, Masumi Iwai-Takano ${ }^{1,2}$, Takashi Igarashi $^{1}$, Hiroharu Shinjo ${ }^{1}$, \\ Keiichi Ishida ${ }^{1}$, Shinya Takase ${ }^{1}$ \& Hitoshi Yokoyama ${ }^{1}$
}

Myocardial fibrosis, as detected by late gadolinium enhancement (LGE) magnetic resonance imaging (MRI), is related to mortality after aortic valve replacement (AVR). This study aimed to determine whether LGEMRI predicts improvement in global longitudinal strain (GLS) after AVR in patients with severe aortic stenosis (AS). Twenty-nine patients with severe AS who were scheduled to undergo AVR were enrolled. Two-dimensional echocardiography and contrast-enhanced MRI were performed before AVR. GLS and LGEcore (g: > 5SD of normal area), LGEgray (g: 2-5SD), and LGEcore+gray (g) were measured. One year after AVR, GLS were examined by echocardiography to assess improvement in LV function. Preoperatively, GLS correlated with LGEcore $(g)\left(r^{2}=0.14, p<0.05\right)$, LGEgray $(g)\left(r^{2}=0.32\right.$, $p<0.01)$ and LGEcore+gray $(g)\left(r^{2}=0.36, p<0.01\right)$. LGEcore was significantly lower in patients with improved GLS after AVR (GLS 1year $\geq-19.9 \%$ ) compared to those with no improvement (1.34 g vs. $4.70 \mathrm{~g}, \mathrm{p}<0.01)$. LGE predicts improvement in LV systolic function after AVR.

Aortic stenosis (AS) remains therapeutic challenge especially in elderly patients. Left ventricular (LV) myocardial fibrosis is associated with progression of LV hypertrophy, which compensates for pressure overload in patients with AS. Myocardial fibrosis is classified as focal fibrosis or diffuse fibrosis, with the latter being an early phenomenon preceding the former ${ }^{1}$. LV myocardial advanced fibrosis, especially focal fibrosis or scars, reportedly correlates with LV systolic dysfunction, and the severity of fibrosis is known to be associated with a poor late prognosis ${ }^{2}$. In some cases, LV dysfunction and heart failure further progress after aortic valve replacement (AVR). Therefore, the optimal timing for AVR needs to be determined while considering the grade of LV myocardial fibrosis. While myocardial biopsy is the gold standard for detecting myocardial fibrosis, its general applicability is limited due to the invasiveness of the procedure.

Cardiac magnetic resonance imaging (MRI) is widely used for assessment of myocardial fibrosis ${ }^{1}$. Late gadolinium enhancement (LGE) MRI is a useful method for detecting focal myocardial fibrosis ${ }^{1}$. Myocardial fibrosis detected by LGE has been reported to correlate with mortality in patients with AS during a median follow up of 2.9 years after $\mathrm{AVR}^{3}$.

Several studies have reported that global longitudinal strain (GLS), an index of LV systolic function assessed by echocardiography, is reduced even in AS patients with preserved LV ejection fraction $(\mathrm{EF})^{4}$. Impaired GLS is known to correlate with AS severity, increased left ventricular mass index (LVMI) ${ }^{5}$, and all-cause mortality in patients with $\mathrm{AS}^{6}$. However, few studies have examined which preoperative examinations predict improvement in GLS after AVR.

This study aimed to examine whether LGE MRI predicts improvement in GLS after AVR in patients with severe AS.

\footnotetext{
${ }^{1}$ Department of Cardiovascular Surgery, Fukushima Medical University, Fukushima, Japan. ${ }^{2}$ These authors contributed equally: Tsuyoshi Fujimiya and Masumi Iwai-Takano. *email: tuyoshif@fmu.ac.jp
} 


\begin{tabular}{|c|c|}
\hline Age, yrs & $73(66-78)$ \\
\hline Men, n (\%) & $15(52)$ \\
\hline Height, $\mathrm{cm}$ & $155.4(148.2-161.7)$ \\
\hline Body weight, kg & $55.2(51.8-60.5)$ \\
\hline Body surface area, $\mathrm{m}^{2}$ & $1.50(1.41-1.60)$ \\
\hline Body mass index, $\mathrm{kg} / \mathrm{m}^{2}$ & $22.8(19.8-25.0)$ \\
\hline \multicolumn{2}{|l|}{ NYHA functional class, $\mathbf{n}(\%)$} \\
\hline $\mathrm{I}$ & $20(69)$ \\
\hline II & $8(28)$ \\
\hline III & $1(3)$ \\
\hline IV & 0 \\
\hline \multicolumn{2}{|l|}{ Symptoms, n (\%) } \\
\hline Dyspnea & $9(31)$ \\
\hline Chest pain & $4(14)$ \\
\hline Syncope & $3(10)$ \\
\hline \multicolumn{2}{|l|}{ Risk factors, n (\%) } \\
\hline Hypertension & $19(66)$ \\
\hline Diabetes mellitus & $4(14)$ \\
\hline Hyperlipidemia & $16(55)$ \\
\hline Current smoker & $5(17)$ \\
\hline \multicolumn{2}{|l|}{ History/comorbidity, n (\%) } \\
\hline Chronic kidney disease & $7(24)$ \\
\hline Cerebral vascular disease & $3(10)$ \\
\hline Chronic obstructive pulmonary disease & $3(10)$ \\
\hline \multicolumn{2}{|l|}{ Medication, n (\%) } \\
\hline$\beta$ blocker & $5(17)$ \\
\hline ACE inhibitor & $2(7)$ \\
\hline ARB & $15(52)$ \\
\hline Anti-aldosterone & $2(7)$ \\
\hline Diuretics & $2(7)$ \\
\hline Brain natriuretic peptide, $\mathrm{pg} / \mathrm{ml}$ & $85.0(39.1-183.0)$ \\
\hline $\mathrm{eGFR}, \mathrm{ml} / \mathrm{min} / 1.73 \mathrm{~m}^{2}$ & $68.0(59.0-74.0)$ \\
\hline Logistic Euro score, \% & $5.13(2.54-6.41)$ \\
\hline
\end{tabular}

Table 1. Patient baseline characteristics $(n=29)$. Continuous variables are expressed as median (interquartile range). NYHA: New York Heart Association, ACE: angiotensin converting enzyme, ARB: angiotensin II receptor blocker, GFR: estimated glomerular filtration rate.

\section{Results}

Baseline characteristics. Table 1 summarized preoperative baseline characteristics of the 29 patients (age, 73 years; $52 \%$ male) included in this study. Nine patients presented with symptoms of heart failure, and 16 patients presented with symptoms of AS. In this cohort, patients had several atherosclerotic risk factors (hypertension, diabetes mellitus, hyperlipidemia, and/or current smoking).

Table 2 shows echocardiographic parameters at baseline. All patients had high-gradient severe AS. LVEF was well-preserved at $65.7 \%$, while GLS was reduced at $-16.5 \%$. LV hypertrophy was observed, but no severe diastolic dysfunction with increased LA pressure was noted.

The parameters of myocardial fibrosis by MRI were showed no severe myocardial fibrosis. As shown in Fig. 2A, significant correlations were observed between GLS and LGEcore $(\mathrm{g})\left(\mathrm{r}^{2}=0.14, \mathrm{p}<0.05\right)$, LGEgray (g) $\left(\mathrm{r}^{2}=0.32, \mathrm{p}<0.01\right)$ and LGEcore+gray $(\mathrm{g})\left(\mathrm{r}^{2}=0.36, \mathrm{p}<0.01\right)$.

Relationships between myocardial fibrosis and imaging parameters. The fibrosis index (FI) obtained from myocardial biopsy specimens of 13 patients was 5.3\% (interquartile range [IQR] 2.8-16.0). FI correlated with LGEcore $(\mathrm{g})\left(\mathrm{r}^{2}=0.38, \mathrm{p}<0.05\right)$ and LGEcore+gray $(\mathrm{g})\left(\mathrm{r}^{2}=0.37, \mathrm{p}<0.05\right)$, but not LGEgray $(\mathrm{g})$ (Fig. 2B). FI strongly correlated with GLS $\left(\mathrm{r}^{2}=0.46, \mathrm{p}<0.05\right)$ (Fig. 2C).

Follow-up echocardiography after AVR. There was no all-cause death or hospitalization due to heart failure at one year after AVR. We examined echocardiography in 26 patients; reasons for not performing follow-up echocardiography included patient refusal, cost of echocardiography, and other socioeconomical reasons. The results of comparisons of echocardiographic parameters before and after AVR are summarized in Table 3. After AVR, aortic valve function was significantly improved in terms of peak velocity ( 4.73 to $2.55 \mathrm{~m} / \mathrm{s}$ ), mean pressure gradient (50.5 to $14.1 \mathrm{mmHg}$ ), aortic valve area $\left(0.65\right.$ to $\left.1.47 \mathrm{~cm}^{2}\right)$, and valvulo-arterial impedance (Zva) $\left(5.46\right.$ to $\left.4.48 \mathrm{mmHg} / \mathrm{ml} / \mathrm{m}^{2}\right)$. There were no paravalvular leakage. Regression of LV hypertrophy and a significant improvement in GLS were observed after AVR ( $-16.9 \%$ to $-19.9 \%)$. 


\begin{tabular}{|c|c|}
\hline \multicolumn{2}{|l|}{ Echocardiography } \\
\hline IVS, mm & $13.1(11.1-14.3)$ \\
\hline $\mathrm{PW}, \mathrm{mm}$ & $12.9(11.4-13.5)$ \\
\hline LVDd, mm & $41.7(37.4-45.5)$ \\
\hline LVDs, mm & $25.4(21.9-28.7)$ \\
\hline LVEDV, ml & $62.8(54.1-77.1)$ \\
\hline LVESV, ml & $21.5(17.2-28.7)$ \\
\hline LV ejection fraction, $\%$ & $65.7(61.9-68.5)$ \\
\hline LVMI, $\mathrm{g} / \mathrm{m}^{2}$ & $123.2(113.0-148.6)$ \\
\hline LAVI, $\mathrm{ml} / \mathrm{m}^{2}$ & $37.4(25.7-48.8)$ \\
\hline $\mathrm{E} / \mathrm{A}$ & $0.63(0.51-0.82)$ \\
\hline $\mathrm{e}^{\prime}, \mathrm{cm} / \mathrm{sec}$ & $4.9(4.4-6.2)$ \\
\hline $\mathrm{E} / \mathrm{e}^{\prime}$ & $12.4(9.4-19.5)$ \\
\hline Peak velocity, $\mathrm{m} / \mathrm{s}$ & $4.72(4.30-5.25)$ \\
\hline Mean PG, mmHg & $51.0(41.4-68.1)$ \\
\hline Aortic valve area, $\mathrm{cm}^{2}$ & $0.67(0.57-0.79)$ \\
\hline $\mathrm{Zva}, \mathrm{mmHg} / \mathrm{ml} / \mathrm{m}^{2}$ & $5.40(4.53-6.50)$ \\
\hline 2D-GLS, $\%$ & $-16.5(-18.2-14.2)$ \\
\hline \multicolumn{2}{|l|}{ MRI } \\
\hline LGEcore, $\mathrm{g}$ & $3.0(1.2-6.7)$ \\
\hline LGEcore, \% & $3.9(1.2-8.4)$ \\
\hline LGEcore, $\mathrm{g} / \mathrm{m}^{2}$ & $2.5(0.7-5.8)$ \\
\hline LGEgray, g & $10.8(7.3-17.8)$ \\
\hline LGEgray, \% & $11.4(8.0-20.9)$ \\
\hline LGEgray, $\mathrm{g} / \mathrm{m}^{2}$ & $8.0(4.6-13.0)$ \\
\hline LGEcore+gray, g & $15.0(9.5-22.7)$ \\
\hline LGEcore+gray, \% & $14.3(10.9-28.5)$ \\
\hline LGEcore+gray, g/m ${ }^{2}$ & $10.6(5.9-17.7)$ \\
\hline
\end{tabular}

Table 2. Preoperative echocardiographic and MRI parameters $(n=29)$. Continuous variables are expressed as median (interquartile range). IVS: interventricular septal thickness, PW: posterior wall thickness, LVDd: left ventricular end-diastolic diameter, LVDs: left ventricular end-systolic diameter, LVEDV: left ventricular end-diastolic volume, LVESV: left ventricular end-systolic volume, LVMI: left ventricular mass index, LAVI: left atrium volume index, PG: pressure gradient, GLS: global longitudinal strain, LGE: late gadolinium enhancement, BSA: body surface area.

We divided the 26 patients who underwent follow-up echocardiography according to median postoperative GLS: the improvement group $(\geq-19.9 \% ; \mathrm{n}=14)$ and the non-improvement group $(<-19.9 \% ; \mathrm{n}=12)$. The comparisons of patient characteristics, echocardiographic parameters, and MRI parameters between the two groups are shown in Table 4.

No significant differences were observed in age, implanted valve size, and blood pressure between the improvement and non-improvement groups. Preoperative echocardiographic parameters did not differ between the two groups. Postoperatively, however, significant improvements were observed in LV hypertrophy (interventricular septal thickness [IVS] and posterior wall thickness [PW]) and LV diastolic function (left ventricular mass index [LVMI] and e') in the improvement group compared to the non-improvement group.

LGEcore and LGEcore+gray were lower in the improvement group compared to the non-improvement group. LGEgray did not differ between the two groups.

In the univariate analysis, LGEcore (g), LGEcore (\% of LV) and LGEcore (g/BSA) were significant predictors of GLS improvement after AVR (LGEcore [g]: $\beta=0.446, p=0.011$; LEGcore [\% of LV]: $\beta=0.452, p=0.020$; LGEcore $[\mathrm{g} / \mathrm{BSA}$ ]: $\beta=0.417, \mathrm{p}=0.034$ ) (Table 5). On the other hand, no preoperative echocardiographic parameters including GLS, LVMI, wall thickness, diastolic indices, hypertension, diabetes and medication predicted improvement in GLS. In the multivariate analysis, LGEcore $(\mathrm{g})$ was found to be an independent predictor of postoperative improvement in GLS $(\beta=0.446, \mathrm{p}=0.022)$ (Table 5).

In the ROC analysis, the area under the curve was 0.81 for predicting postoperative GLS improvement $(\geq-19.9 \%$ ) by LGEcore (g), with a cut-off value of $2.86 \mathrm{~g}$ (sensitivity, $78.6 \%$; specificity, $83.3 \%$ ) (Fig. 3A).

Figure $3 \mathrm{~B}$ shows changes in GLS before and after AVR for each patient. Patients with low LGEcore $(<2.86 \mathrm{~g})$ showed improved GLS after AVR compared to those with high LGEcore ( $\geq 2.86 \mathrm{~g})$.

\section{Discussion}

In this study, we investigated whether preoperative LGEs could predict improvement in GLS after AVR in patients with preserved LVEF and reduced GLS. The major findings are as follows: 1) Preoperative examinations revealed significant correlations among GLS, LGEs, and FI; 2) One year after AVR, GLS was improved in a manner dependent on preoperative LGEcore; and 3) LGEcore can predict postoperative improvement in GLS with a 


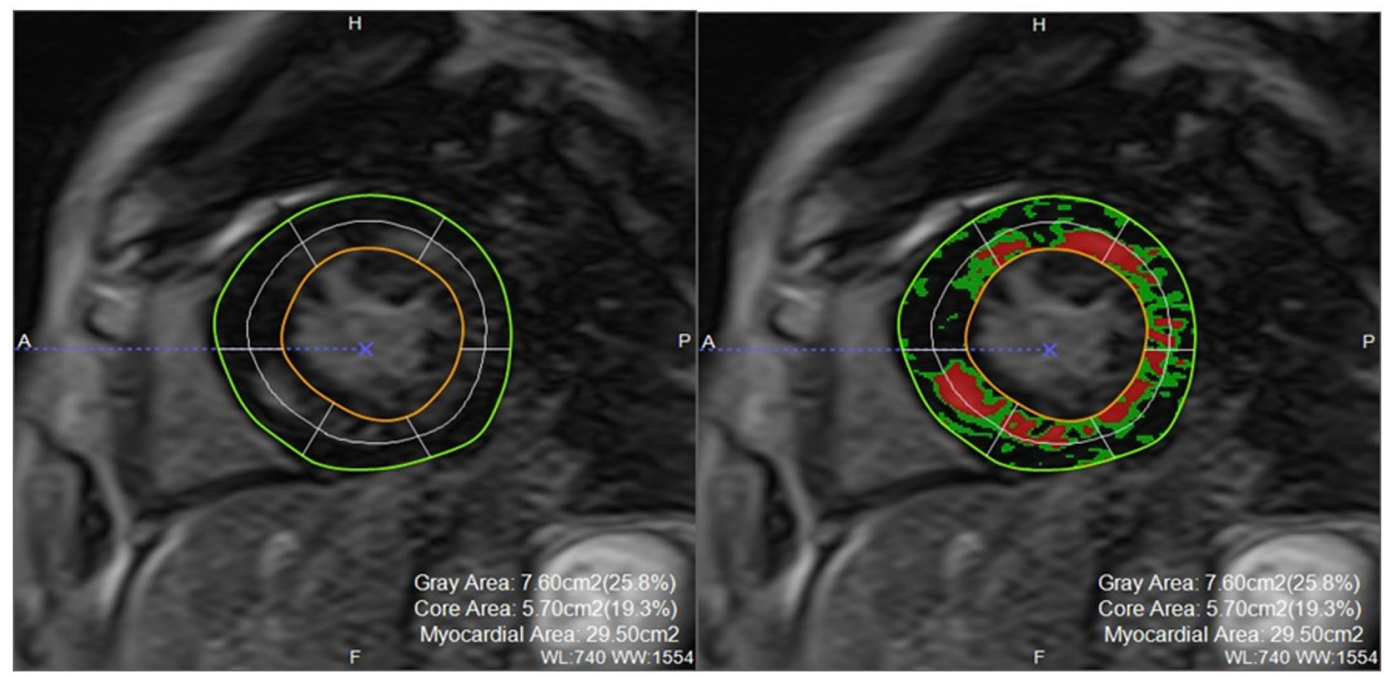

Figure 1. Measurement of LGE by MRI. LGEcore, LGEgray, and LGEcore+gray were calculated as areas with the above-threshold signal intensity in the ROI ( $\geq 5$ SD for LGEcore and 2-5 SD for LGEgray compared to the normal area). ROI: region of interest. LGE: late gadolinium enhancement.

cut-off value of $2.86 \mathrm{~g}$. These findings suggest that myocardial fibrosis as detected by LGE predicts improvement in GLS after AVR.

Relationships among GLS, LGEs, and FI. In the present study, GLS correlated with LGEcore+gray. On the other hand, the correlation with LGEcore was weak. LGEgray didn't correlate with FI. Because interstitial diffuse fibrosis, interstitial edema and myocardial hypertrophy weren't detected as FI, LGEgray containing them didn't correlate with FI. GLS correlated well with LGEcore+ gray including focal and diffuse fibrosis because GLS reflected the state of the whole myocardium.

Microscopic changes in LV are characterized by cardiomyocyte hypertrophy and extracellular matrix expansion in patients with AS. These conditions are caused by either focal replacement fibrosis (scar) or reactive and interstitial diffuse fibrosis ${ }^{2,3,7-11}$. According to previous studies, LGEcore and LGEgray reflect focal fibrosis and diffuse mild interstitial fibrosis, respectively ${ }^{12}$.

In a previous study, histological findings suggested improved GLS in patients with mild fibrosis ${ }^{9}$. Lee et al. also reported that native T1 values by cardiac MRI as an index of diffuse interstitial fibrosis correlated with GLS ${ }^{13}$. Reduction of GLS correlates with several factors such as myocardial fibrosis ${ }^{14}$, pressure overload, and obesity ${ }^{15}$, improved after AVR if fibrosis was mild in previous report ${ }^{9}$. Although the main cause of GLS impairment is still unknown, as well as the prospect for GLS improvement after AVR, it suggests that myocardium of patients with severe AS contains reversible and irreversible fibrosis.

GLS is reduced in symptomatic patients with severe AS, and a decrease in GLS is a predictor of all-cause mortality ${ }^{6}$. GLS is also a predictor of future major adverse cardiac events in asymptomatic patients with severe AS and preserved $\mathrm{LVEF}^{16}$. Thus, assessing GLS is clinically important in patients with potential systolic dysfunction and preserved LVEF. In the present cohort study, patients had preserved LVEF with a slight decrease in GLS, and myocardial specimens showed mild fibrosis compared to severity of fibrosis in the previous reports ${ }^{13}$. However, given that not all patients showed improved GLS after AVR, predictors of GLS improvement after AVR need to be investigated further.

Prediction of improvement in GLS after AVR. In the present study, LGEcore, but not LGEgray or LGEcore+gray, was found to be a predictor of GLS improvement after AVR. While LGEgray (i.e., mild interstitial fibrosis) can be reversible, LGEcore (i.e., focal fibrosis) is unlikely to improve after AVR. Thus, our findings suggest that the degree of focal fibrosis before AVR is a determining factor for GLS improvement after AVR in patients with severe AS.

A previous cohort study reported that LGE did not improve significantly 9 months after AVR 9 . Since focal fibrosis doesn't improve, the decrease in diffuse fibrosis contributes to the improvement of postoperative contractility. A recent prospective observational cohort study reported that focal fibrosis (scars) as detected by LGE does not resolve, while diffuse fibrosis and myocardial hypertrophy as assessed by extracellular volume (ECV) show significant regression after AVR in patients with symptomatic severe $\mathrm{AS}^{7}$. However, it remains unclear as to which type of LV myocardial fibrosis (i.e., focal or diffuse) plays an important role in persistent systolic dysfunction after AVR.

To the best of our knowledge, this is the first study to evaluate whether LGE as an index of focal fibrosis and/or diffuse fibrosis could predict improvement in GLS one year after AVR. Preoperative GLS strongly correlated with LGEgray, but weakly correlated with LGEcore. On the other hand, LGEcore was found to be a predictor of GLS improvement after AVR. The use of different thresholds, i.e., > 5 standard deviation (SD) for LGEcore and 2-5SD 


\begin{tabular}{|l|l|l|l|}
\hline & Pre-AVR & Post-AVR & P value \\
\hline IVS, $\mathrm{mm}$ & $13.1(11.5-14.2)$ & $10.8(9.0-12.2)$ & $<0.001$ \\
\hline PW, $\mathrm{mm}$ & $13.0(11.6-13.4)$ & $10.1(9.0-11.6)$ & $<0.001$ \\
\hline LVDd, mm & $41.5(37.3-46.3)$ & $43.0(37.4-45.2)$ & 0.76 \\
\hline LVDs, mm & $25.6(22.0-29.0)$ & $25.7(21.0-29.6)$ & 0.80 \\
\hline LVEDV, ml & $62.9(54.4-78.7)$ & $63.6(56.3-79.7)$ & 0.88 \\
\hline LVESV, $\mathrm{ml}$ & $21.9(17.3-31.2)$ & $23.2(19.5-30.2)$ & 0.74 \\
\hline LV ejection fraction, \% & $65.6(61.8-68.7)$ & $65.6(57.6-67.4)$ & 0.34 \\
\hline LVMI, g/m ${ }^{2}$ & $123.2(113.1-142.3)$ & $92.9(81.1-110.0)$ & $<0.001$ \\
\hline LAVI, $\mathrm{ml} / \mathrm{m}^{2}$ & $35.5(20.8-48.7)$ & $30.6(24.4-39.0)$ & 0.28 \\
\hline E/A & $0.62(0.51-0.82)$ & $0.91(0.73-1.14)$ & 0.014 \\
\hline e', cm/sec & $5.2(4.5-6.4)$ & $7.5(5.9-9.6)$ & 0.001 \\
\hline E/e' & $12.1(8.8-14.5)$ & $9.7(8.1-14.2)$ & 0.38 \\
\hline Peak velocity, m/s & $4.73(4.18-5.35)$ & $2.55(2.44-3.01)$ & $<0.001$ \\
\hline Mean PG, mmHg & $50.5(39.4-70.0)$ & $14.1(11.5-17.1)$ & $<0.001$ \\
\hline Aortic valve area, $\mathrm{cm}^{2}$ & $0.65(0.56-0.76)$ & $1.47(1.20-1.75)$ & $<0.001$ \\
\hline Zva, $\mathrm{mmHg} / \mathrm{ml} / \mathrm{m}^{2}$ & $5.46(4.98-6.51)$ & $4.48(3.37-5.04)$ & 0.001 \\
\hline 2D-GLS, \% & $-16.9(-18.9-14.2)$ & $-19.9(-22.1-17.9)$ & 0.004 \\
\hline
\end{tabular}

Table 3. Comparison of pre- and postoperative echocardiographic parameters $(n=26)$. Continuous variables are expressed as median (interquartile range). IVS: interventricular septal thickness, PW: posterior wall thickness, LVDd: left ventricular end-diastolic diameter, LVDs: left ventricular end-systolic diameter, LVEDV: left ventricular end-diastolic volume, LVESV: left ventricular end-systolic volume, LVMI: left ventricular mass index, LAVI: left atrium volume index, PG: pressure gradient, GLS: global longitudinal strain.

for LGEgray, allowed us to detect potential systolic dysfunction with preserved LVEF (LGEgray), and to predict improvement in GLS (LGEcore) after AVR.

Clinical implication. Recent therapeutic strategies for asymptomatic severe AS include AVR, which is recommended only when LVEF is less than $50 \%{ }^{17}$. However, severe AS patients with preserved LVEF already has LV myocardial fibrosis ${ }^{18}$. In patients with extensive focal fibrosis, myocardial damage persists even if LV afterload is decreased by AVR. Thus, myocardial fibrosis needs to be evaluated noninvasively in order to predict prognosis after AVR in a clinical setting. Since focal fibrosis as detected by LGEcore $(<2.86 \mathrm{~g})$ is an independent predictor of GLS improvement after AVR, surgical therapy should be considered before patients develop irreversible LV dysfunction.

Study limitations. This study has several limitations. First, this study was conducted at a single center. Second, we excluded patients with chronic kidney disease (CKD) because of a contraindication to contrast-enhanced MRI. Thus, the results of the present study may not apply to patients with CKD, which is a common disorder in elderly patients. Other methods to assess LV myocardial fibrosis, e.g., ECV by MRI ${ }^{19,20}$, should be considered. Third, we assessed GLS by 2D echocardiography, not 3D echocardiography. A significant correlation has been reported between 2D GLS and 3D GLS in patients with AS, and 3D GLS as well as 2D GLS are reportedly predictors of major adverse cardiac events ${ }^{13}$. Forth, the myocardial specimens were endomyocardial and not transmural which raised issue of representability.

In conclusion, this prospective observational study demonstrated that LGEcore predicts improvement in GLS after AVR in patients with severe AS and preserved LVEF.

\section{Methods}

Study design and patient recruitment. This prospective observational study was conducted in 29 patients with severe AS who underwent AVR from January 2014 to July 2017. Severe AS was defined as an aortic valve area $<1.0 \mathrm{~cm}^{2}$, peak aortic valve velocity $>4.0 \mathrm{~m} / \mathrm{s}$, and mean pressure gradient $>40 \mathrm{mmHg}^{21}$. Exclusion criteria were patients with concomitant severe aortic regurgitation, moderate to severe mitral regurgitation, and a previous history of ischemic heart disease, atrial fibrillation, left bundle branch block, or CKD (eGFR $<30 \mathrm{ml} / \mathrm{min} / 1.73 \mathrm{~m}^{2}$ is a contraindication to gadolinium enhanced-MRI $)^{22}$.

All patients underwent echocardiography and MRI prior to AVR. Myocardial biopsy specimens were collected intraoperatively from 13 patients who obtained informed consent. We evaluated relationships among the parameters of echocardiography and MRI and myocardial specimens. Echocardiography was also performed one year after AVR to assess the correlation between preoperative LGE and postoperative GLS improvement.

This study was approved by the institutional review board of Fukushima Medical University and was conducted in compliance with the principles of the Declaration of Helsinki. All patients provided written informed consent.

Echocardiography. We performed transthoracic echocardiography to assess aortic valve function and LV systolic and diastolic function using the Acuson SC2000 ${ }^{\mathrm{TM}}$ system (SIEMENS: Mountain View, CA, USA) with a $4-\mathrm{MHz}$ transducer ${ }^{23,24}$. Echocardiographic parameters included LV wall thickness and dimension, LV volume 


\begin{tabular}{|c|c|c|c|}
\hline & $\begin{array}{l}\text { Improvement group } \\
(\mathrm{n}=14)\end{array}$ & $\begin{array}{l}\text { Non-improvement } \\
\text { group }(n=12)\end{array}$ & P value \\
\hline Age, yrs & $73.0(65.5-78.3)$ & $72.5(58.8-75.8)$ & 0.71 \\
\hline Hypertension, $\mathrm{n}(\%)$ & $9(64)$ & $8(67)$ & 0.90 \\
\hline Diabetes, n (\%) & $2(14)$ & $2(17)$ & 0.87 \\
\hline \multicolumn{4}{|l|}{ Medication } \\
\hline$\beta$ blocker, n (\%) & $1(7)$ & $3(25)$ & 0.21 \\
\hline ACE inhibitor, n (\%) & $1(7)$ & $1(8)$ & 0.91 \\
\hline ARB, n (\%) & $6(43)$ & $6(50)$ & 0.72 \\
\hline Anti-aldosterone, n (\%) & 0 & $1(8)$ & 0.27 \\
\hline Diuretics, n (\%) & 0 & $1(8)$ & 0.27 \\
\hline \multicolumn{3}{|l|}{ Implanted valve size } & 0.26 \\
\hline $19 \mathrm{~mm}, \mathrm{n}$ & 7 & 4 & \\
\hline $21 \mathrm{~mm}, \mathrm{n}$ & 5 & 7 & \\
\hline $23 \mathrm{~mm}, \mathrm{n}$ & 2 & 0 & \\
\hline $27 \mathrm{~mm}, \mathrm{n}$ & 0 & 1 & \\
\hline Preoperative sBP, mmHg & $117(102-136)$ & $121(117-131)$ & 0.71 \\
\hline Postoperative sBP, mmHg & $126(118-136)$ & $131(114-140)$ & 0.56 \\
\hline \multicolumn{4}{|c|}{ Preoperative echocardiography } \\
\hline IVS, $\mathrm{mm}$ & $13.2(12.1-14.7)$ & $13.0(10.5-14.0)$ & 0.63 \\
\hline $\mathrm{PW}, \mathrm{mm}$ & $12.8(11.6-13.6)$ & $13.1(11.5-13.5)$ & 0.71 \\
\hline LVDd, mm & $40.9(37.5-42.2)$ & $45.5(37.0-50.3)$ & 0.13 \\
\hline LVDs, mm & $24.4(19.5-27.7)$ & $26.1(22.8-32.0)$ & 0.19 \\
\hline LVEDV, ml & $60.8(51.7-70.4)$ & $69.5(57.6-90.2)$ & 0.11 \\
\hline LVESV, ml & $19.2(16.0-26.5)$ & $25.3(17.6-31.7)$ & 0.25 \\
\hline LV ejection fraction, $\%$ & $66.0(60.5-70.1)$ & $65.4(62.4-67.2)$ & 0.90 \\
\hline LVMI, $\mathrm{g} / \mathrm{m}^{2}$ & $118.9(108.7-137.8)$ & $127.7(115.2-164.6)$ & 0.37 \\
\hline LAVI, $\mathrm{ml} / \mathrm{m}^{2}$ & $37.4(20.8-48.7)$ & $33.5(22.2-58.6)$ & 0.98 \\
\hline $\mathrm{E} / \mathrm{A}$ & $0.62(0.51-0.71)$ & $0.68(0.51-1.18)$ & 0.35 \\
\hline $\mathrm{e}^{\prime}, \mathrm{cm} / \mathrm{sec}$ & $5.3(4.5-6.2)$ & $5.2(4.5-7.0)$ & 0.94 \\
\hline $\mathrm{E} / \mathrm{e}^{\prime}$ & $10.6(8.4-14.5)$ & $12.6(10.1-18.2)$ & 0.32 \\
\hline Peak velocity, $\mathrm{m} / \mathrm{s}$ & $4.73(4.18-5.38)$ & $4.75(4.07-5.43)$ & 0.98 \\
\hline Mean PG, mmHg & $52.0(40.8-76.8)$ & $50.5(38.1-68.6)$ & 0.78 \\
\hline Aortic valve area, $\mathrm{cm}^{2}$ & $0.64(0.54-0.77)$ & $0.66(0.56-0.81)$ & 0.67 \\
\hline $\mathrm{SVi}, \mathrm{ml} / \mathrm{m}^{2}$ & $34.5(25.6-35.6)$ & $31.4(24.6-36.0)$ & 0.61 \\
\hline $\mathrm{Zva}, \mathrm{mmHg} / \mathrm{ml} / \mathrm{m}^{2}$ & $5.81(4.10-7.07)$ & $5.41(4.99-6.27)$ & 0.76 \\
\hline 2D-GLS, \% & $-17.7(-20.5-14.9)$ & $-15.2(-18.1-12.7)$ & 0.18 \\
\hline \multicolumn{4}{|c|}{ Postoperative echocardiography } \\
\hline IVS, $\mathrm{mm}$ & $9.7(8.7-11.8)^{*}$ & $11.5(9.9-13.4)$ & 0.041 \\
\hline $\mathrm{PW}, \mathrm{mm}$ & $9.5(8.6-10.6)^{*}$ & $11.1(9.9-13.0)$ & 0.036 \\
\hline LVDd, $\mathrm{mm}$ & $43.0(38.0-44.4)$ & $41.8(37.4-45.4)$ & 0.86 \\
\hline LVDs, $\mathrm{mm}$ & $24.9(21.4-29.6)$ & $25.7(20.1-29.7)$ & 0.82 \\
\hline LVEDV, ml & $62.9(55.1-79.7)$ & $65.3(55.9-81.9)$ & 0.63 \\
\hline LVESV, ml & $24.8(17.6-30.6)$ & $22.1(20.4-29.2)$ & 0.94 \\
\hline LV ejection fraction, $\%$ & $65.2(57.5-67.4)$ & $65.5(61.4-68.4)$ & 0.82 \\
\hline LVMI, $\mathrm{g} / \mathrm{m}^{2}$ & $82.2(74.5-101.2)^{*}$ & $102.7(92.4-127.6)^{*}$ & 0.036 \\
\hline LAVI, $\mathrm{ml} / \mathrm{m}^{2}$ & $29.8(22.9-34.1)$ & $37.2(24.1-52.2)$ & 0.30 \\
\hline $\mathrm{E} / \mathrm{A}$ & $0.97(0.79-1.25)^{*}$ & $0.9(0.6-1.1)$ & 0.32 \\
\hline $\mathrm{e}^{\prime}, \mathrm{cm} / \mathrm{sec}$ & $8.5(6.9-10.6)^{*}$ & $6.0(5.1-8.0)$ & 0.011 \\
\hline $\mathrm{E} / \mathrm{e}^{\prime}$ & $9.2(7.1-11.9)$ & $11.6(9.3-15.8)$ & 0.044 \\
\hline Peak velocity, $\mathrm{m} / \mathrm{s}$ & $2.6(2.5-3.1)^{*}$ & $2.5(2.3-3.0)^{*}$ & 0.53 \\
\hline Mean PG, mmHg & $15.7(12.1-17.1)^{*}$ & $13.0(11.2-17.7)^{*}$ & 0.49 \\
\hline Aortic valve area, $\mathrm{cm}^{2}$ & $1.26(1.12-1.61)^{*}$ & $1.60(1.32-1.79)^{*}$ & 0.28 \\
\hline $\mathrm{SVi}, \mathrm{ml} / \mathrm{m}^{2}$ & $35.1(32.7-40.3)^{\dagger}$ & $33.3(24.7-40.1)$ & 0.33 \\
\hline $\mathrm{Zva}, \mathrm{mmHg} / \mathrm{ml} / \mathrm{m}^{2}$ & $4.11(3.39-4.78)^{*}$ & $4.42(3.15-5.58)$ & 0.33 \\
\hline 2D-GLS, \% & $-22.1(-22.4-20.3)^{*}$ & $-17.6(-18.7-13.4)$ & 0.001 \\
\hline \multicolumn{4}{|l|}{ Preoperative MRI } \\
\hline LGEcore, $\mathrm{g}$ & $1.34(0.81-2.98)$ & $4.70(2.99-9.00)$ & 0.005 \\
\hline \multicolumn{4}{|l|}{ Continued } \\
\hline
\end{tabular}




\begin{tabular}{|l|l|l|l|}
\hline & $\begin{array}{l}\text { Improvement group } \\
(\mathbf{n}=\mathbf{1 4})\end{array}$ & $\begin{array}{l}\text { Non-improvement } \\
\text { group }(\mathbf{n = 1 2})\end{array}$ & P value \\
\hline LGEcore, \% & $1.40(1.10-4.43)$ & $5.50(2.03-8.50)$ & 0.036 \\
\hline LGEcore, g/m ${ }^{2}$ & $0.91(0.59-2.23)$ & $3.39(1.83-6.28)$ & 0.015 \\
\hline LGEgray, g & $8.72(4.40-13.48)$ & $10.8(10.1-18.8)$ & 0.12 \\
\hline LGEgray, \% & $11.0(7.23-14.3)$ & $10.4(8.35-20.6)$ & 0.46 \\
\hline LGEgray, g/m ${ }^{2}$ & $5.61(3.10-8.67)$ & $7.55(6.13-12.7)$ & 0.12 \\
\hline LGEcore+gray, g & $9.62(6.84-15.24)$ & $18.8(13.4-26.2)$ & 0.013 \\
\hline LGEcore+gray, \% & $12.9(8.53-16.1)$ & $15.8(12.0-26.7)$ & 0.16 \\
\hline LGEcore+gray, g/m ${ }^{2}$ & $6.49(4.50-10.5)$ & $11.6(8.19-18.1)$ & 0.023 \\
\hline
\end{tabular}

Table 4. Comparison of echocardiographic and MRI parameters between groups with or without GLS improvement. ACE: angiotensin converting enzyme, ARB: angiotensin II receptor blocker, sBP: systolic blood pressure, IVS: interventricular septal thickness, PW: posterior wall thickness, LVDd: left ventricular enddiastolic diameter, LVDs: left ventricular end-systolic diameter, LVEDV: left ventricular end-diastolic volume, LVESV: left ventricular end-systolic volume, LVMI: left ventricular mass index, LAVI: left atrium volume index, PG: pressure gradient, SVi: stroke volume index, GLS: global longitudinal strain, LGE: late gadolinium enhancement, BSA: body surface area. ${ }^{*} \mathrm{p}<0.01$ vs. preoperative echocardiography. ${ }^{\dagger} \mathrm{p}<0.05 \mathrm{vs}$. preoperative echocardiography.

\begin{tabular}{|l|l|l|l|l|}
\hline \multirow{2}{*}{} & \multicolumn{2}{|l|}{ Univariate analysis } & \multicolumn{2}{l|}{ Multivariate analysis } \\
\cline { 2 - 5 } & $\boldsymbol{\beta}$ & P value & $\boldsymbol{\beta}$ & P value \\
\hline Preoperative GLS & 0.264 & 0.10 & & \\
\hline LGEcore (g) & 0.446 & 0.011 & 0.446 & 0.022 \\
\hline LGEcore (\% of LV) & 0.452 & 0.020 & & \\
\hline LGEcore (g/BSA) & 0.417 & 0.034 & & \\
\hline LGEcore+gray (g) & 0.319 & 0.056 & & \\
\hline LGEcore+gray (\% of LV) & 0.313 & 0.120 & & \\
\hline LGEcore+gray (g/BSA) & 0.276 & 0.173 & & \\
\hline
\end{tabular}

Table 5. Multivariate analysis to predict postoperative improvement in GLS. GLS: global longitudinal strain, LGE: late gadolinium enhancement, LV: left ventricle, BSA: body surface area, ACE: angiotensin converting enzyme, ARB: angiotensin II receptor blocker.

and LVEF, left atrium volume index (LAVI), peak early filling (E velocity) and late filling (A velocity) using pulsed-wave Doppler images, peak early diastolic velocity (mean E' of lateral and septal wall) using tissue Doppler images, E/e' and AS indices (aortic valve area, peak velocity, mean pressure gradient [MPG], and Zva). Zva was defined as the ratio of estimated LV systolic pressure (the sum of systolic arterial pressure [SAP] and MPG) to stroke volume index $(\mathrm{SVi}): \mathrm{Zva}=(\mathrm{SAP}+\mathrm{MPG}) / \mathrm{SVi}^{25}$. $\mathrm{LV}$ mass index was calculated by the cube formula in the parasternal long-axis view ${ }^{23}$.

2D-GLS was examined by 2D speckle tracking echocardiography using the SC2000 workplace system $\mathrm{VVI}^{\mathrm{TM}}$ (SIEMENS: Mountain View, CA, USA). We assessed endocardial GLS as the average of GLSs in apical 2-, 3-, and 4-chamber views ${ }^{26}$.

Cardiac MRI. Cardiac MRI was performed on a 1.5-T scanner (Vantage Titan ${ }^{\mathrm{TM}}$ : Canon Medical Systems, Otawara, Japan) according to the standard LGE protocol ${ }^{27}$. Ten minutes before image acquisition, $1.0 \mathrm{M}$ gadobutrol (Gadovist ${ }^{\mathrm{TM}}$ : Bayer, Berlin, Germany), a gadolinium-based contrast agent, was administered systemically to patients with $\mathrm{eGFR} \geq 30 \mathrm{ml} / \mathrm{min} / 1.73 \mathrm{~m}^{2}$.

Cardiac MRI was analyzed using a post-processing workstation (Ziostation2 ${ }^{\mathrm{TM}}$ : Ziosoft, Tokyo, Japan). The contours of the LV endocardium and epicardium were traced semi-automatically in short-axis slices. The region of interest (ROI) was selected within the remote reference myocardium to set the $\mathrm{SD}^{28}$. All LGE measurements were performed by the author (T.F), and $95 \%$ intraclass correlation coefficient was 0.858 . We evaluated LGEs as parameters of fibrosis, calculated on the workstation as areas with the above-threshold signal intensity compared to the remote reference myocardium in the ROI (LGEcore: > 5SD; LGEgray: 2-5 SD; LGEcore+gray: LGEcore plus LGEgray) (Fig. 1) ${ }^{12,29}$. These indices were expressed as absolute amounts (g), percentage of myocardial mass (\% of LV), and amounts corrected by body surface area (g/BSA).

Intraoperative biopsy. Intraoperative myocardial biopsy specimens were taken from 13 of the 29 patients. An endomyocardial specimen roughly $8 \mathrm{~mm}^{3}$ in volume was obtained from the basal muscular septum $2 \mathrm{~cm}$ below the outflow tract following aortic valve resection. All specimens were preserved in $20 \%$ formalin, embedded in paraffin, cut into $5-\mu \mathrm{m}$-thick sections, and stained with Elastica-Masson stain. The myocardial muscle and fibrous tissue was observed at a magnification of $100 \mathrm{x}^{30}$. The FI was defined as the ratio (in percentage) of fibrosis tissue to the total myocardial field using Image $\mathrm{J}^{31}$. For each patient, FI was quantified in five different fields representative of all myocardial samples. 
A
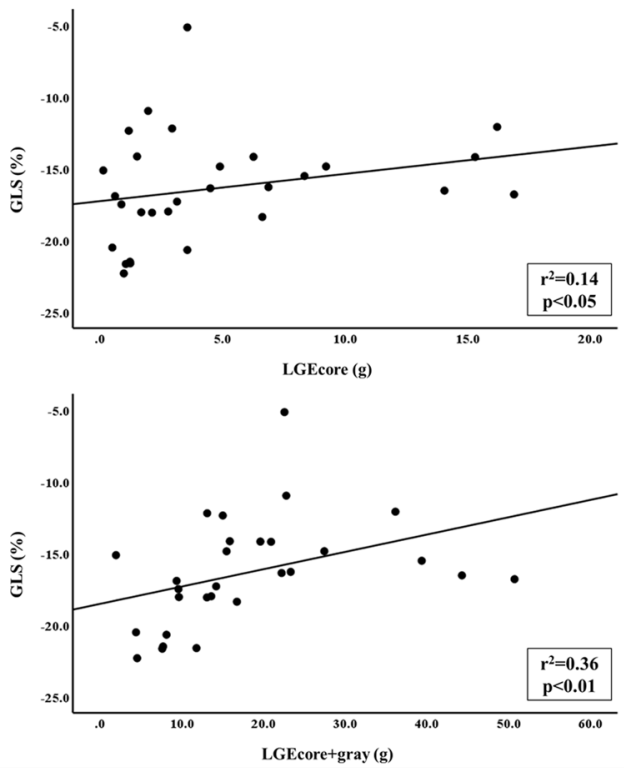

$\mathbf{B}$
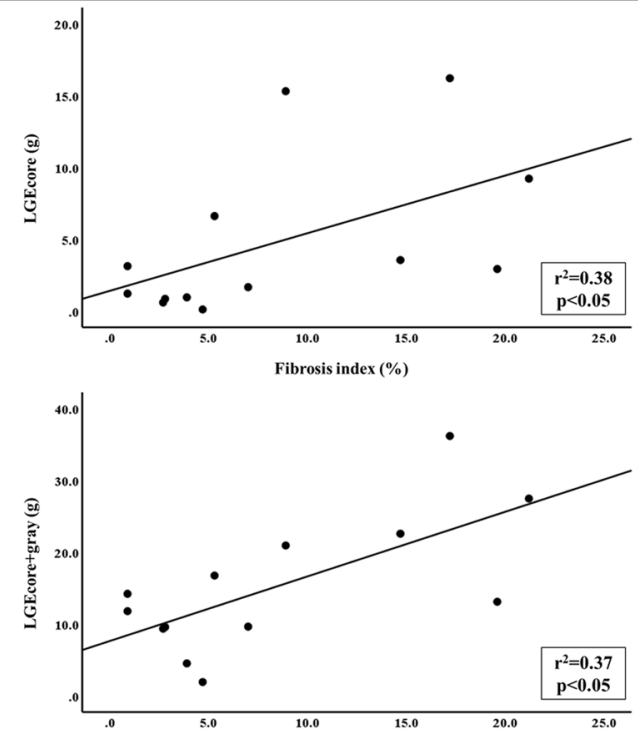

Fibrosis index $(\%)$

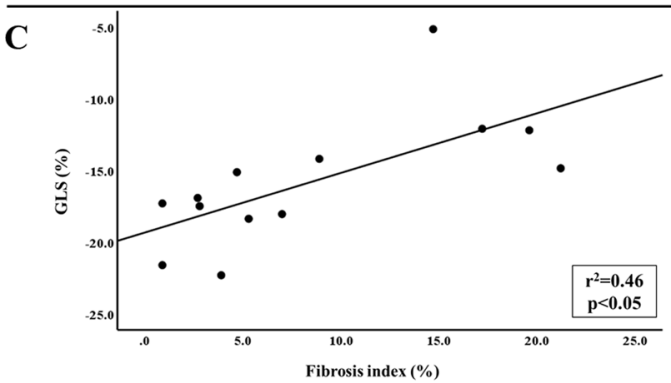

Figure 2. Correlation between GLS, LGE and FI. (A) GLS was significantly correlated with LGEcore (g) $\left(\mathrm{r}^{2}=0.14, \mathrm{p}<0.05\right)$, LGEgray $(\mathrm{g})\left(\mathrm{r}^{2}=0.32, \mathrm{p}<0.01\right)$ and LGEcore+gray $(\mathrm{g})\left(\mathrm{r}^{2}=0.36, \mathrm{p}<0.01\right)$. (B) LGEcore (g) $\left(\mathrm{r}^{2}=0.38, \mathrm{p}<0.05\right)$ and LGEcore+gray $(\mathrm{g})\left(\mathrm{r}^{2}=0.37, \mathrm{p}<0.05\right)$, but not LGEgray, was significantly correlated with the fibrosis index. (C) GLS was significantly correlated with the fibrosis index $\left(\mathrm{r}^{2}=0.46\right.$, $\mathrm{p}<0.05)$.

Relationships among echocardiography, MRI, and myocardial specimens at baseline and follow-up. We evaluated relationships among preoperative GLS by echocardiography, LGEcore, LGEgray, and LGEcore+ gray by MRI, and FI derived from myocardial specimens. 

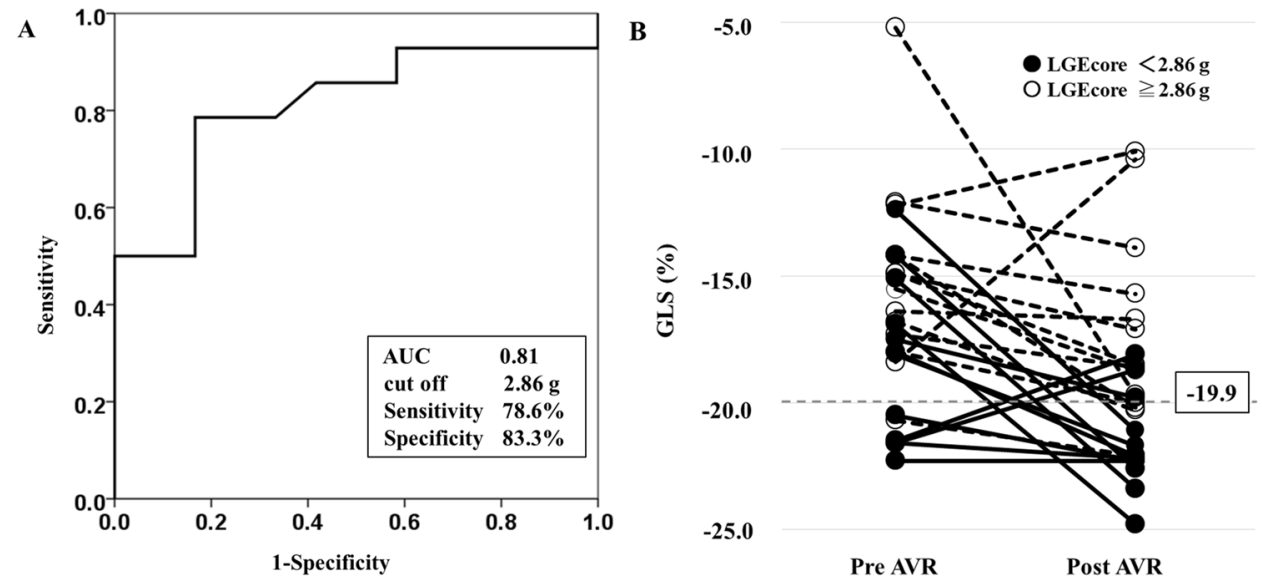

Figure 3. Receiver-Operating Characteristics (ROC) Curve Analysis for Prediction of GLS Improvement after AVR. (A) In the ROC analysis, the area under the curve was 0.81 for predicting postoperative GLS improvement $(\geq-19.9 \%)$ by LGEcore, with a cut-off value of $2.86 \mathrm{~g}$ (sensitivity, $78.6 \%$; specificity, $83.3 \%$ ). (B) Patients with low LGEcore $(<2.86 \mathrm{~g})$ showed improvement in GLS after AVR compared to those with high LGEcore $(\geq 2.86 \mathrm{~g})$. Black circle: LGEcore $<2.86 \mathrm{~g}$, white circle: LGEcore $\geq 2.86 \mathrm{~g}$.

Patients underwent echocardiography one year after AVR and were divided into the following two groups according to GLS improvement: the improvement group (postoperative GLS greater than or equal to median) and the non-improvement group (post-operative GLS less than median).

Pre- and postoperative echocardiographic parameters and LGEs were compared between the improvement group and the non-improvement group in order to assess whether it is possible to predict improvement in GLS after AVR. Moreover, multivariate analysis was performed to determine which parameters are independent predictors of GLS improvement.

Statistical analysis. Statistical analyses were performed using SPSS ${ }^{\mathrm{TM}}$ software version 23 (IBM, Armonk, New York). Categorical variables were expressed as percentages. All continuous variables were expressed as a median (IQR). Comparisons between the improvement and non-improvement group were assessed by the Mann-Whitney $U$ test for non-normally distributed variables and the chi-square test for categorical variables. On the other hand, the comparison of pre- and postoperative results was assessed by Wilcoxon paired t test. Log transformation was used to normalize the distribution of preoperative GLS, LGEcore, and LGEcore+gray. Multiple linear regression was used to predict postoperative improvement in GLS based on preoperative GLS, LGEcore, and LGEcore+gray. For each parameter, log-converted values were used for multivariate analysis (i.e., $\mathrm{x}:[\log \mathrm{x}] /[\mathrm{SD}$ of $\log \mathrm{x}])$.

Received: 10 March 2019; Accepted: 4 October 2019;

Published online: 30 October 2019

\section{References}

1. Bing, R. et al. Imaging and impact of myocardial fibrosis in aortic stenosis. J Am Coll Cardiol Img 12, 283-96 (2019).

2. Azevedo, C. F. et al. Prognostic significance of myocardial fibrosis quantification by histopathology and magnetic resonance imaging in patients with severe aortic valve disease. J Am Coll Cardiol 56, 278-87 (2010).

3. Barone-Rochette, G. et al. Prognostic significance of LGE by CMR in aortic stenosis patients undergoing valve replacement. J Am Coll Cardiol 64, 144-54 (2014).

4. Kusunose, K. et al. Incremental prognostic value of left ventricular global longitudinal strain in patients with aortic stenosis and preserved ejection fraction. Circ Cardiovasc Imaging 7, 938-45 (2014).

5. Dinh, W. et al. Reduced global longitudinal strain in association to increased left ventricular mass in patients with aortic valve stenosis and normal ejection fraction: a hybrid study combining echocardiography and magnetic resonance imaging. Cardiovasc Ultrasound 8, 29 (2010).

6. $\mathrm{Ng}$, A. C. T. et al. Left ventricular global longitudinal strain is predictive of all-cause mortality independent of aortic stenosis severity and ejection fraction. Eur Heart J Cardiovasc Imaging 19, 859-67 (2018).

7. Treibel, T. A. et al. Reverse myocardial remodeling following valve replacement in patients with aortic stenosis. J Am Coll Cardiol 71, 860-71 (2018).

8. Dweck, M. R. et al. Midwall fibrosis is an independent predictor of mortality in patients with aortic stenosis. J Am Coll Cardiol 58, 1271-9 (2011).

9. Weidemann, F. et al. Impact of myocardial fibrosis in patients with symptomatic severe aortic stenosis. Circulation 120, 577-84 (2009).

10. Herrmann, S. et al. Low-gradient aortic valve stenosis myocardial fibrosis and its influence on function and outcome. J Am Coll Cardiol 58, 402-12 (2011).

11. Yarbrough, W. M., Mukherjee, R., Ikonomidis, J. S., Zile, M. R. \& Spinale, F. G. Myocardial remodeling with aortic stenosis and after aortic valve replacement: mechanisms and future prognostic implications. J Tjorac. Cardiovasc Surg 143, 656-64 (2012).

12. Roes, S. D. et al. Infarct tissue heterogeneity assessed with contrast-enhanced MRI predicts spontaneous ventricular arrhythmia in patients with ischemic cardiomyopathy and implantable cardioverter-defibrillator. Circ Cardiovasc Imaging 2, 183-90 (2009).

13. Lee, S. P. et al. Assessment of diffuse myocardial fibrosis by using MR imaging in asymptomatic patients with aortic stenosis. Radiology 274, 359-69 (2015) 
14. Hoffmann, R., Altiok, E., Friedman, Z., Becker, M. \& Frick, M. Myocardial deformation imaging by two-dimensional speckletracking echocardiography in comparison to late gadolinium enhancement cardiac magnetic resonance for analysis of myocardial fibrosis in severe aortic stenosis. Am J Cardiol 114, 1083-8 (2014).

15. Ávila-Vanzzini, N. et al. Echocardiographic and histologic correlations in patients with severe aortic stenosis: Influence of overweight and obesity. J Cardiovasc Ultrasound 24, 303-11 (2016).

16. Nagata, Y. et al. Prognostic value of LV deformation parameters using 2D and 3D speckle-tracking echocardiography in asymptomatic patients with severe aortic stenosis and preserved LV ejection fraction. J Am Coll Cardiol Img 8, 235-45 (2015).

17. Nishimura, R. A. et al. 2014 AHA/ACC guideline for the management of patients with valvular heart disease: executive summary: a report of the American College of Cardiology/American Heart Association task force on practice guidelines. J Am Coll Cardiol 63, 2438-88 (2014).

18. Hein, S. et al. Progression from compensated hypertrophy to failure in the pressure-overloaded human heart: structural deterioration and compensatory mechanisms. Circulation 107, 984-91 (2003).

19. Chin, C. W. L. et al. Myocardial fibrosis and cardiac decompensation in aortic stenosis. J Am Coll Cardiol Img 10, 1320-33 (2017).

20. Moon, J. C. et al. Myocardial T1 mapping and extracellular volume quantification: A Society for Cardiovascular Magnetic Resonance (SCMR) and CMR working group of the European Society of Cardiology consensus statement. J Cardiovasc Magn Reson 15, 92 (2013).

21. Baumgartner, H. et al. Recommendations on the echocardiographic assessment of aortic valve stenosis: A focused update from the European Association of Cardiovascular Imaging and the American Society of Echocardiography. J Am Soc Echocardiogr 30, 372-92 (2017).

22. Kribben, A. et al. Nephrogenic systemic fibrosis pathogenesis, diagnosis, and therapy. J Am Coll Cardiol 53, 1621-8 (2009).

23. Lang, R. M. et al. Recommendations for cardiac chamber quantification by echocardiography in adults: An update from the American Society of Echocardiography and the European Association of Cardiovascular Imaging. J Am Soc Echocardiogr 28, 1-39 (2015).

24. Nagueh, S. F. et al. Recommendations for the evaluation of left ventricular diastolic function by echocardiography: An update from the American Society of Echocardiography and the European Association of Cardiovascular Imaging. J Am Soc Echocardiogr 29, 277-314 (2016).

25. Hachicha, Z., Dumesnil, J. G. \& Pibarot, P. Usefulness of the valvuloarterial impedance to predict adverse outcome in asymptomatic aortic stenosis. J Am Coll Cardiol 54, 1003-11 (2009).

26. Reisner, S. A. et al. Global longitudinal strain: a novel index of left ventricular systolic function. J Am Soc Echocardiogr 17, 630-633 (2004).

27. Crouch, G. et al. Early effects of transcatheter aortic valve implantation and aortic valve replacement on myocardial function and aortic valve hemodynamics: Insights from cardiovascular magnetic resonance imaging. J Thorac Cardiovasc Surg 149, 462-70 (2015).

28. Spartera, M., Damascelli, A., Mozes, F., De Cobelli, F. \& La Canna, G. Three-dimensional speckle tracking longitudinal strain is related to myocardial fibrosis determined by late-gadolinium enhancement. Int J Cardiovasc Imaging 33, 1351-60 (2017).

29. Gao, P. et al. Prediction of arrhythmic events in ischemic and dilated cardiomyopathy patients referred for implantable cardiac defibrillator: evaluation of multiple scar quantification measures for late gadolinium enhancement magnetic resonance imaging. Circ Cardiovasc Imaging 5, 448-56 (2012).

30. Milano, A. D. et al. Prognostic value of myocardial fibrosis in patients with severe aortic valve stenosis. J Thorac Cardiovasc Surg 144, $830-7(2012)$.

31. Schneider, C. A., Rasband, W. S. \& Eliceiri, K. W. NIH Image to ImageJ: 25 years of image analysis. Nat Methods 9, 671-5 (2012).

\section{Acknowledgements}

We thank Dr. Tetsuya Ohira, Professor of Department of Epidemiology, Fukushima Medical University, for his advice on statistical analysis.

\section{Author contributions}

T.F., M.I.-T., T.I. and H.Y. designed the research; T.F., M.I.-T., T.I., H.S. and K.I. performed the research; T.F. and M.I.-T. analyzed the data; T.F., M.I.-T., and H.Y. wrote the paper; S.T. and H.Y. provided clinical advice.

\section{Competing interests}

The authors declare no competing interests.

\section{Additional information}

Correspondence and requests for materials should be addressed to T.F.

Reprints and permissions information is available at www.nature.com/reprints.

Publisher's note Springer Nature remains neutral with regard to jurisdictional claims in published maps and institutional affiliations

Open Access This article is licensed under a Creative Commons Attribution 4.0 International License, which permits use, sharing, adaptation, distribution and reproduction in any medium or format, as long as you give appropriate credit to the original author(s) and the source, provide a link to the Creative Commons license, and indicate if changes were made. The images or other third party material in this article are included in the article's Creative Commons license, unless indicated otherwise in a credit line to the material. If material is not included in the article's Creative Commons license and your intended use is not permitted by statutory regulation or exceeds the permitted use, you will need to obtain permission directly from the copyright holder. To view a copy of this license, visit http://creativecommons.org/licenses/by/4.0/.

(C) The Author(s) 2019 